\title{
Transformer Fault Diagnosis based on RBF Neural Network
}

\section{Xicheng Teng}

\author{
Beijing University of Chemical and Technology, Beijing, 100029, China. \\ tengxicheng@126.com
}

Keywords: neural network; transformer; fault diagnosis; training model.

\begin{abstract}
With respect to the transformer fault diagnosis, this paper proposed a fault diagnosis model based on RBF neural network, realized RBF neural network based on center vector of gauss basis function, the weight calculation method of RBF based on Kalman filtering, the training and testing algorithm of the data classification model is given. The proposed method of the transformer fault diagnosis based on RBF neural network is discussed in detail. Because the modularized structure is adopted and each sub-model is only used to recognize one fault, the difficulty of training model is reduced, it is more important that the ability and application flexibility of the fault diagnosis are improved obviously. Research results show that the proposed method has strong robustness and high accuracy.
\end{abstract}

\section{Introduction}

Transformer is an indispensable power equipment in power system, and its reliable and stable work plays a crucial role in the power grid. The voltage responsible for the work for the voltage conversion, power distribution and power transmission[1]. However, the accuracy of the traditional fault diagnosis method can not meet the diagnostic requirements of the present stage due to the fault of the power equipment and the complex multilaterality of the power failure. We know that the reliable, accurate and timely monitoring of transformers is very important for people's lives and property. Therefore, it is necessary to study a convenient, reliable and accurate method of transformer fault diagnosis. With the rapid development of artificial intelligence, people tend to use intelligent means to carry out fault diagnosis. Neural network is a research focus in the field of artificial intelligence. It has excellent parallel processing, learning, memory, nonlinear mapping, adaptive and robustness, making it very suitable for the field of fault diagnosis of transformers [2-5]. Among them, BP neural network has a wide range of applications in the field of transformer fault diagnosis. However, the number of hidden layers in BP neural network is not easy to be determined and the weight training is difficult. In this paper, transformer fault diagnosis model based on RBF neural network is constructed, and Kalman filter algorithm is used to set the weight of RBF neural network. The specific design method will be described in detail in the following sections of this article.

\section{RBF Neural Network}

RBF neural network has been successfully applied to nonlinear function approximation and time series analysis, Data classification, pattern recognition, information processing, image processing, system modeling, control and fault diagnosis [6,7].

Kalman filter, first proposed by Kalman in 1960, is a linear minimum variance estimation algorithm that is recursive and uses state space method to design filters in the time domain. Therefore, Kalman filter is suitable for multidimensional Estimation of random processes. The weight estimation of RBF neural network is a multi-dimensional random estimation process and also a core part of the RBF training process. In this paper, the gain of RBF neural network $g(k)$ is used to improve the selection of RBF weights $\omega_{l i}^{0}$. The specific process is as follows:

$$
g(k)=\frac{P(k-1) \mathrm{z}^{T}(k)}{\lambda+\mathrm{z}(k) P(k-1) \mathrm{z}^{T}(k)}
$$




$$
P(k)=\frac{1}{\lambda}[P(k-1)-g(k) \mathrm{z}(\mathrm{k}) P(k-1)]
$$

Taking $g(k)$ into equation (2) to obtain the connection vector:

$$
\omega_{i i}(k)=\omega_{i}(k-1)+\varepsilon_{j}(k) g^{T}(k), 1 \leq l i \leq M
$$

Where $\varepsilon_{j}$ is the setting error.

\section{Design of RBF Neural Network for The Fault Diagnosis Model}

\subsection{The Structure of Fault Diagnosis Model}

As the transformer breaks down when there is mainly gas $\mathrm{H}_{2}, \mathrm{CH}_{4}, \mathrm{C}_{2} \mathrm{H}_{2}, \mathrm{CO}, \mathrm{CO}_{2}$. In this paper, the percentage of the total volume of five gases in the transformer decomposition gas is taken $\left(\mathrm{H}_{2}, \mathrm{CH}_{4}, \mathrm{C}_{2} \mathrm{H}_{2}, \mathrm{C}_{2} \mathrm{H}_{4}, \mathrm{C}_{2} \mathrm{H}_{6}\right)$ as the input of the RBF neural network, so the node of the input layer is determined to be five. This paper presents a modular diagnostic model based on Radial Basis Function (RBF) neural network for transformer fault diagnosis, as shown in Figure 1.

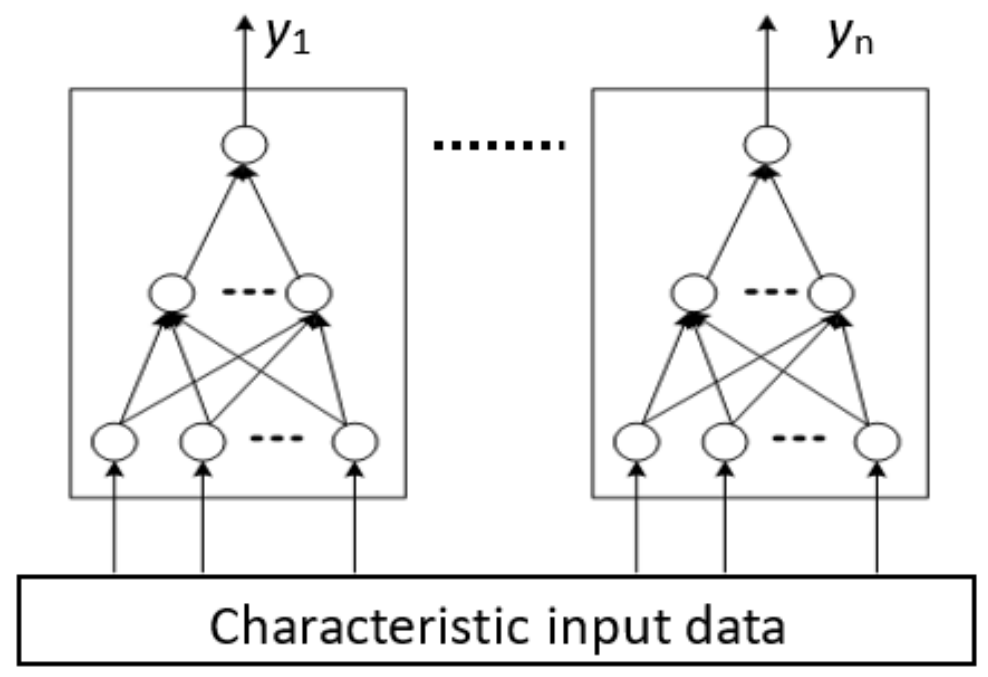

Fig 1. The model of transformer fault

In the model of Figure 1, each module or sub-model consists of a multi-input single-output RBF neural network, corresponding to a fault condition of the transformer.

Here set the numerical size between 0 and 1 indicates the corresponding degree of failure, the closer to 1 indicates the greater the corresponding fault severity. Table 1 shows the RBF neural network output corresponding to the transformer fault type.

Table 1. The model output status

\begin{tabular}{|c|c|c|c|c|c|}
\hline & Sub-model(1) & Sub-model(2) & Sub-model(3) & Sub-model(4) & Sub-model(5) \\
\hline No fault & 1 & 0 & 0 & 0 & 0 \\
\hline Low temperature overheating & 0 & 1 & 0 & 0 & 0 \\
\hline High temperature overheating & 0 & 0 & 1 & 0 & 0 \\
\hline Low-energy discharge & 0 & 0 & 0 & 1 & 0 \\
\hline High-energy discharge & 0 & 0 & 0 & 0 & 1 \\
\hline
\end{tabular}

\subsection{The Algorithm Transformer Fault Diagnosis Model}

The proposed RBF neural network fault diagnosis model algorithm steps are as follows:

1) network initialization

(1) input layer to the hidden layer of the weight matrix:

$\omega_{l i}^{0}, 1 \leq i \leq N, 1 \leq l \leq L$, usually $=1$;

(2) hidden layer to the output layer of the weight matrix: 
$\omega_{j l}^{0}, 1 \leq j \leq M$, the random function to choose $\omega_{j l}^{0}$;

(3) randomly select the initial inverse correlation matrix $P(0)_{L \times L}$;

(4) set the error $\varepsilon_{j}=0.01$ or set the number of iterations to be terminated;

(5) forgetting factor: $0 \leq \lambda \leq 1$;

2) Select the center vector $C_{i}$ of RBF network by the method of Section 1.1;

3) Calculate Gaussian parameters

$$
\alpha_{l}=\frac{\beta^{2}}{N}\left(2 M_{t}\right)^{-\beta / N}
$$

In formula (5), $\mathrm{N}$ is the input sample matrix dimension; $M_{t}$ is the number of samples; $\beta$ is the confidence level;

4) Calculate the RBF output according to equation (6) below

$$
z_{l}(t)=\exp \left[-\frac{\sum_{i=1}^{N}\left(x_{i}(t)-c_{i}\right)^{2}}{2 \alpha_{l}^{2}}\right], 1 \leq l \leq L
$$

5) Start iteration $k=1$;

6) calculate the output layer of each node error

$y_{i}(k)=z(k) \omega_{l i}^{T}(k-1)$;

$\varepsilon_{i}(k)=d_{i}(k)-y_{i}(k), 1 \leq j \leq M$;

7) Get the new connection vector by using Section 1.2

$\omega_{i i}(k)=\omega_{i}(k-1)+\varepsilon_{j}(k) g^{T}(k), 1 \leq l i \leq M$

8) Calculate cumulative error energy

$$
J(k)=\lambda J(k-1)+\frac{1}{2} \sum_{j=1}^{M} \varepsilon_{j}^{2}(k), 1 \leq j \leq M
$$

9) If $J(k) \geq \varepsilon$, then $k=k+1$, return 4), otherwise perform 10);

10) End the operation

\subsection{Training Gesture Recognition Model}

The sub-model structure is selected as RBF-based three-layer neural network with five input nodes and one output node. This article selected 6 hidden nodes, then the final sub-model structure is 5-61. Data collected in these five states represent a no-fault condition, a medium to low temperature overheating condition, a high temperature overheat, a low-energy discharge condition, and a highenergy discharge condition, respectively. The collected 40 groups of data are divided into two categories, of which 25 for network training, 15 for network testing, due to the large number of data, a list of a set of no-fault training samples and a set of no-fault test samples, such as table 2,3 shows:

Table 2. Training samples

\begin{tabular}{|l|l|}
\hline No falut & $(0.645,0.114,0.558,0.641,0.811)$ \\
\hline
\end{tabular}

Table 3. Testing samples

\begin{tabular}{|c|c|}
\hline No fault & $(0.524,0.108,0.5033,0.641,0.798)$ \\
\hline
\end{tabular}

If $J(k) \geq \varepsilon, k<$ the maximum number of iterations is reached, the training sample data is input cyclically; if $J(k)<\varepsilon, k=$ the maximum number of iterations is reached, the loop is stopped, Output sub-model parameters $\alpha_{l}, c_{l}, \omega_{j}$. 
The training samples of 25 groups are taken as the input of 5 sub-models respectively. The output of each corresponding RBF sub-model is shown in Table 1. There are five sub-models sub_M1 $\left(c_{1}, \alpha_{1}, \omega_{1}\right)$, sub_M2 $\left(c_{2}, \alpha_{2}, \omega_{2}\right)$, sub_M3 $\left(c_{3}, \alpha_{3}, \omega_{3}\right)$, sub_M4 $\left(c_{4}, \alpha_{4}, \omega_{4}\right), \operatorname{Sub}$ M5 $\left(c_{5}, \alpha_{5}, \omega_{5}\right)$ of the parameters, considering the length of the article, not listed here one by one

The following test samples were used to test the five sub-models obtained, the first group corresponds to a no-fault state, and the last four groups correspond to a faulty state. In other words, the sub model sub_M1 $\left(c_{1}, \alpha_{1}, \omega_{1}\right)$ should output $\left(\begin{array}{lllll}1 & 0 & 0 & 0 & 0\end{array}\right)$ and the sub model sub_M2 $\left(c_{2}, \alpha_{2}, \omega_{2}\right)$ should be $\left(\begin{array}{lllll}0 & 1 & 0 & 0 & 0\end{array}\right)$ while testing the submodels with these 15 sets of data. The submodels $\left(\begin{array}{lllll}0 & 0 & 1 & 0 & 0\end{array}\right)$ is output from sub_M3 $\left(c_{3}, \alpha_{3}, \omega_{3}\right),\left(\begin{array}{lllll}0 & 0 & 0 & 1 & 0\end{array}\right)$ from sub model sub_M4 $\left(c_{4}, \alpha_{4}, \omega_{4}\right)$ and $\left(\begin{array}{lllll}0 & 0 & 0 & 0 & 1\end{array}\right)$ from sub model sub_M5 $\left(c_{5}, \alpha_{5}, \omega_{5}\right)$. Figure 3-5 shows the output histogram of the five sub-models tested with test data.

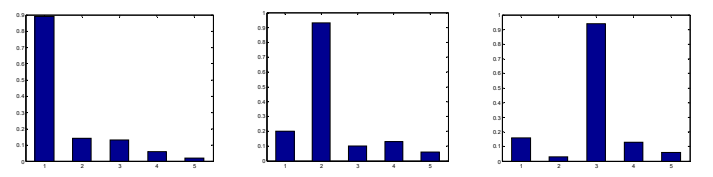

Fig 2. The output of sub_M1 $\left(c_{1}, \alpha_{1}, \omega_{1}\right) \ldots$ sub_M3 $\left(c_{3}, \alpha_{3}, \omega_{3}\right)$

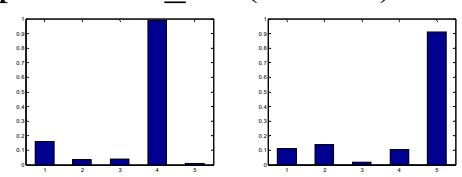

Fig 3. The output of sub_M4 $\left(c_{4}, \alpha_{4}, \omega_{4}\right)$, sub_M5 $\left(c_{5}, \alpha_{5}, \omega_{5}\right)$

In this paper, the output of each RBF sub-model structure is set to be 1 when the output value of each RBF sub-model structure is greater than 0.5, and 0 when less than 0.5. From Fig. 2 and Fig. 3, we can see that the five sub-models can effectively identify the fault condition of the same transformer, and the five sub-models constructed by the test data can be tested with the accuracy of up to $100 \%$. Therefore, The fault model is valid.

\section{Summary}

The proposed fault diagnosis model based on transformer construction and training method has the characteristics of flexible model construction, easy training, fast convergence, low power consumption and so on. Reasonable choice of radial basis function parameters can promote the model convergence, shorten the model training time and improve the model attitude recognition ability. The model test results show that the proposed method is feasible and the fault diagnosis model established is effective.

\section{References}

[1]. Tang Yongbo, Guihua Hua, Ouyang Wei. Transformer Fault Diagnosis Based on Relative Reconstruction Contribution [J]. High Voltage Technology, 2012: 2964-2971.

[2]. He Zhenyu, Jin Lianwen. Activity recognition from acceleration data using AR model representation and SVM [C]. IEEE International Conference on Machine Learning and Cybernetics, 2008: 2245-2250.

[3]. Chen Xiaoqing, Liu Juemin, Huang Yingwei, et al. Transformer fault diagnosis based on improved artificial fish swarm optimization rough set algorithm [J]. High Voltage Technology, 2012: 1403-1409.

[4]. Prakash S, Parvathi R. Qualitative approach for quantitative association rule mining using fuzzy set [J]. Journal of Computation Information Systems, 2011, 17 (6): 1879-1885.

[5]. Mao Yu-xing, Chen Tong-bin, Shi Bo-le. Efficient method for mining multiple-level and generalized association rule [J]. Journal of Software, 2011,22 (12): 2965-2980. 
[6]. Pedro Vicente Jover Rodriguez, Marian Negrea, Antero Arkkio. A simplified scheme for induction motor condition monitoring [J]. Mechanical systems and signal Processing, 2008, 22: 1216-1236.

[7]. V. N. Ghate; S. V. Dudul. Design of optimal MLP and RBF neural network classifier for fault diagnosis of three phase induction motor [J]. International Journal of Advanced Mechatronic Systems, 2010, 2 (3): 204-216.

[8]. Ye Jian, Ge Lin-Dong, Wu Yue-Xian. An application of improved RBF neural network in modulation recognition. Acta Automatica Sinica, 2007, 33 (6): 652-654. 